\title{
Theory of stellar convection: removing the mixing-length parameter
}

\author{
Stefano Pasetto ${ }^{1}$, Cesare Chiosi ${ }^{2}$, Mark Cropper ${ }^{1}$ and Eva K. Grebel ${ }^{3}$ \\ ${ }^{1}$ Mullard Space Science Laboratory, University College London, Holmbury, St. Mary, \\ Dorking, Surrey RH5 6NT, United Kingdom \\ email: s.pasetto@ucl.ac.uk \\ ${ }^{2}$ Dept. of Physics \& Astronomy "Galileo Galilei", University of Padua, Vicolo \\ dell'Osservatorio, 5, 35141 Padova PD, Italy, \\ ${ }^{3}$ Astronomisches Rechen-Institut, Zentrum für Astronomie der Universität Heidelberg, \\ Mönchhofstr 12-14, 69120 Heidelberg, Germany
}

\begin{abstract}
Stellar convection is customarily described by the mixing-length theory, which makes use of the mixing-length scale to express the convective flux, velocity, and temperature gradients of the convective elements and stellar medium. The mixing-length scale is taken to be proportional to the local pressure scale height, and the proportionality factor (the mixing-length parameter) must be determined by comparing the stellar models to some calibrator, usually the Sun. No strong arguments exist to suggest that the mixing-length parameter is the same in all stars and all evolutionary phases. Because of this, all stellar models in the literature are hampered by this basic uncertainty.

In a recent paper (Pasetto et al. 2014) we presented a new theory that does not require the mixing length parameter. Our self-consistent analytical formulation of stellar convection determines all the properties of stellar convection as a function of the physical behaviour of the convective elements themselves and the surrounding medium. The new theory of stellar convection is formulated starting from a conventional solution of the Navier-Stokes/Euler equations, i.e. the Bernoulli equation for a perfect fluid, but expressed in a non-inertial reference frame co-moving with the convective elements. In our formalism, the motion of stellar convective cells inside convective-unstable layers is fully determined by a new system of equations for convection in a non-local and time-dependent formalism.

We obtained an analytical, non-local, time-dependent solution for the convective energy transport that does not depend on any free parameter. The predictions of the new theory are compared with those from the standard mixing-length paradigm with positive results for atmosphere models of the Sun and all the stars in the Hertzsprung-Russell diagram.
\end{abstract}

Keywords. Sun: fundamental parameters Sun: interior stars: evolution stars: fundamental parameters

\section{Introduction}

The transfer of energy by convection is of paramount importance in all the stars. High-mass stars, roughly for masses $m>1.3 m_{\odot}$ contain fully convective cores, all stars $m \in\left[0.1,100\left[m_{\odot}\right.\right.$ have outer convective envelopes, and finally stars smaller in mass than $m<0.3 m_{\odot}$ are fully convective. Despite its great importance, a satisfactory treatment of stellar convection in stars is still open to debate and a self-consistent treatment of the physics of convective energy transfer is still missing. The ideal goal would be to obtain a set of self-consistent equations, i.e. a set of equations resulting from physical assumptions without ad-hoc parameters to be determined by means of suitable calibrators (usually the Sun). 
It is worth recalling here that while convcetion in the inner regions of a star can be simply reduced to assuming the temperature gradient of the convectively unstable medium equal to the adiabatic value and to suppose that convection can easily carry any amount of energy, in the external regions this is no longer possible and some theory has to be used to predict the temperature gradient in the medium and convective elements and the amount of energy that can be carried by convection (the remaining part being transported by radiation). The most successful theory dealing with the external convection is the mixing-length Theory (MLT) long ago developed by Biermann (1951) and Böhm-Vitense (1958). The MLT is paradigm reference to which every new theory has to be compared with because of its success over decades in which it has been used. In the MLT, the convective elements are supposed to travel a mean-free-path $l_{m}$ (e.g., Kippenhahn et al. 2012). $l_{m}$ which is assumed to be proportional to the natural distance scale $h_{P}$, given by the pressure stratification of the star, the proportionality constant being the mixing-length (ML) parameter $\Lambda_{m}$, defined implicitly as $l_{m} \equiv \Lambda_{m} h_{P}$. The parameter $\Lambda_{m}$ is derived from comparing the theoretical luminosity, radius and effective temperature of a stellar model for the Sun to its observational values.

In a recent paper Pasetto et al. (2014) developed the first theory of stellar convection in which the solar properties are reproduced without making use of free parameters. In the following we will refer to this theory as the scale-free convection (SFC) theory. In this approach, reviewed in the next sections, the authors obtained a solution for the equations governing stellar atmospheres that self-consistently predict the energy transport, luminosities, radii and effective temperatures all along the evolutionary sequence of a star.

\section{A mixing-length free set of equation for stellar atmospheres}

In this section, after a short presentation of the basic stellar equations, we will review the fundamentals of the Pasetto et al. (2014) theory of convection in stellar atmospheres. On purpose, the discussion is kept light and equation-free in order to help the reader to understand the results presented in Section 3 below. The reader interested to any other detail of the new theory is referred to the original paper by Pasetto et al. (2014).

\subsection{Equation of a stellar layer}

The atmosphere of a star is considered as a spherical distribution of gaseous plasma in absence of rotation and magnetic field and in pressure equilibrium against self-gravity. Assuming spherical coordinates $\{r, \theta, \phi\}$ centered on the star's center, any layer of thickness $d r$ and mass $d m$ obeys to the mass conservation equation, $\frac{d m}{d r}=4 \pi r^{2} \rho$, where $\rho$ is the mass density. The layer aloso obeys the Poisson equation linking the density distribution $\rho$ to its gravitational potential $\Phi_{\mathrm{g}}$, i.e. $\Delta_{r} \Phi_{\mathrm{g}}=4 \pi g \rho$ where $\Delta_{r}$ is the Laplacian operator in spherical coordinates. The layer has an equations of motion (EoM) that at equilibrium in spherical symmetry is represented by the Euler equation, $\frac{1}{4 \pi r^{2}} \frac{\partial^{2} r}{\partial t^{2}}=-\frac{\partial P}{\partial m}-\frac{G m}{4 \pi r^{4}}$, where $t$ is the time and $G$ the gravitational constant. Finally, the equation for the energy transfer $\frac{\partial L}{\partial m}=\varepsilon-\varepsilon_{\nu}+\varepsilon_{g}$ has to be considered. In principle, the layer could contain nuclear $\varepsilon=\varepsilon\left(P, T, X_{i}\right)$ and gravitational $\varepsilon_{\mathbf{g}}=\varepsilon_{\mathbf{g}}\left(P, T, X_{i}\right)$ sources of energy, and in some circumstances even the neutrino production and consequent energy losses $\varepsilon_{\nu}=\varepsilon_{\nu}\left(P, T, X_{i}\right)$ could be considered. However, in a stellar atmosphere the production of energy by nuclear, gravitational, and neutrinos can be ignored and the luminosity is simply assumed to be a constant (the luminosity has already been set up to its total value in the deep interiors). Finally, the plasma obeys an equation of state that links pressure $P$, temperature $T$, density $\rho$ and chemical composition of the elements, $X_{i}(i=1, n, n$ the number of 
chemical elements considered) and a suitable relation for the radiative opacity function of the same quantities (in some cases the conductive opacity has to be considered). Last, in a stellar atmosphere, the transport of energy is secured by radiation plus conduction and convection. This environment represents the medium in which any theory of convection has to be framed.

In addition to this we need a numerical procedure to solve all the above equations together with their boundary conditions. We adoptthe code for stellar models written by Hofmeister et al. (1964) and largely modified and updated by the Padua group: (e.g., Chiosi \& Summa 1970) with semiconvection, Bressan et al. (1981) with ballistic convective overshoot from the core, Alongi et al. (1991) with envelope overshoot, Deng et al. (1996a), Deng et al. (1996b) and Salasnich et al. (1999) with turbulent diffusion, finally the many revision and improvements described in Bertelli et al. (1994), Bertelli et al. (1995), Bertelli \& Nasi (2001), Bertelli et al. (2003), Bertelli et al. (2008). In the future we will be implement the new theory of convection also in the twin-code developed independently by (Weiss \& Schlattl 2008). The SFC theory of Pasetto et al. (2014) and the classical MLT are run in parallel so that comparison is possible.

\subsection{The idea for a new theory of stellar convection}

The ideas at the base of the SFC theory are in principle simple. Let us think for example of the upward motion of a convective element. The evolution of a single convective cell can be considered as the sum of the upward motion the expansion. In the MLT only the upward motion is considered. The free-parameter of the MLT stems indeed from the assumptions made to describe the upward motion of the convective elements. Therefore, the only logical alternative in developing a new theory is to consider the expansion of the convective as the main driver of the whole process. To make the upward motion ineffective it is enough to write the equations describing the motion of a convective element in a reference frame co-moving with it. In such a case, all equatiions are referred to convective element and this latter convective is at rest. Pasetto et al. (2014) name $S_{1}$ this comoving reference, to distinguish it from the inertial reference frame centred on the star and named $S_{0}$. In Pasetto et al. (2014) the hydrodynamic equations have been integrated accounting for the non-inertial apparent forces that arise in the treatment of any physical system evolving in $S_{1}$. Under the assumption that viscous terms are much smaller than the inertial ones and the magnetic field is negligible, the potential flow approximation can be adopted and suitably formulated in $S_{1}$ (mathematical formulation in $S_{1}$ is slightly more complicated). In order to keep the equations analytically treatable, Pasetto et al. (2014) limit the analysis to the linear regime. If we limit ourselves to the subsonic regime of the stellar convection, the velocity of a convective element, $\mathbf{v}$, will be much smaller that its expansion rate, $\left\|\frac{d \xi}{d t}\right\| \equiv\|\dot{\xi}\|$, where $\xi$ is the size of the convective elements. Therefore, a linear theory on the small parameter $\varepsilon \equiv \frac{\|\mathbf{v}\|}{\|\dot{\xi}\|} \ll 1$ can be developed. In particular, within the framework of this linear approximation, in the equations governing the evolution of the expansion rate of a convective element, the role of the inertia of the fluid displaced by the motion of the convective element turns out to be important. In contrast, this term has been always neglected in the literature and the evolution of the convective elements was always studied only in relation to its vertical motion (this led indeed to the problem of the mixing-length scale).

As a result of this approach, we obtaine a new system of equations for the energy transfer as a function of the radiative plus conductive flux $\varphi_{\text {rad } \mid \text { cnd }}$, the convective flux $\varphi_{\mathrm{cnv}}$, the average temperature over pressure gradient $\nabla_{e} \equiv\left|\frac{d \ln T}{d \ln P}\right|_{e}$ of the element, and the stellar gradient $\nabla$. Moreover, two extra variables, the mean velocity $\bar{v}$ and the mean 
$\bar{\xi}_{e}$ of the convective elements are obtained as a result of the solution of the system. All these physical quantities are a function of the pressure $P$, temperature $T$, density $\rho$, specific heat at constant pressure $c_{p}$, adiabatic gradient of temperature over pressure $\nabla_{\text {ad }} \equiv\left|\frac{d \ln T}{d \ln P}\right|_{\text {ad }}$, radiative gradient $\nabla_{\mathrm{rad}}$, molecular weight gradient $\nabla_{\mu} \equiv \frac{d \ln \mu}{d \ln P}$, the gravity $g$, the opacity $\kappa$. Finally, all these quantities are a function of the position inside the star and time.

The general form of the system of equations obtained in Pasetto et al. (2014) is:

$$
\left\{\begin{aligned}
\varphi_{\mathrm{rad} / \mathrm{cnd}} & =\frac{4 a c}{3} \frac{T^{4}}{\kappa h_{P} \rho} \nabla \\
\varphi_{\mathrm{rad} / \mathrm{cnd}}+\varphi_{\mathrm{cnv}} & =\frac{4 a c}{3} \frac{T^{4}}{\kappa h_{P} \rho} \nabla_{\mathrm{rad}} \\
\bar{v}^{2} & =\frac{\nabla-\nabla_{e}-\frac{\varphi}{\delta} \nabla_{\mu}}{\frac{3 h P}{2 \delta \bar{\tau}}+\left(\nabla_{e}+2 \nabla-\frac{\varphi}{2 \delta} \nabla_{\mu}\right)} \bar{\xi}_{e} g \\
\varphi_{\mathrm{cnv}} & =\rho c_{P} T\left(\nabla-\nabla_{e}\right) \frac{\bar{v}^{2} \tau}{h_{P}} \\
\frac{\nabla_{e}-\nabla_{\mathrm{ad}}}{\nabla-\nabla_{e}} & =\frac{4 a c T^{3}}{\kappa \rho^{2} c_{P}} \frac{\tau}{\bar{\xi}_{e}^{2}} \\
\bar{\xi}_{e} & =\frac{g}{4} \frac{\nabla}{\frac{3 h P}{2 \delta \bar{v} \tau}+\left(\nabla_{e}+2 \nabla-\frac{\varphi}{\delta} \nabla_{\mu}\right.} \overline{\left.\frac{\varphi}{2 \delta} \nabla_{\mu}\right)} \bar{\chi}
\end{aligned}\right.
$$

where $a$ is the radiation-density constant, and $c$ the speed of light and for the purposes of this paper $\bar{\chi}$ is a function of time linking size to velocity (i.e. a monotonic linear map (a bijection) between time, velocity and size of the convective elements). For any other see Appendix A of Pasetto et al. (2014)).

It is worth remarking here that the SFC theory, although developed in spherical coordinates, does not really depend on it because no assumption is made about the shape of convective elements whwn carrying energy up and down. The elements indeed are not separated from the surrounding medium by a surface (in such a case one should use the Young-Laplace treatment of the surface tension). Our approach differ from the classical physical description in literature (e.g., Tuteja et al. 2010, and references therein), but it agrees with astrophysical 3D-hydrodynamical simulations in which the convection is represented by small volumes moving up and down for a short time†.

This system of equations can be proved to be "closed", i.e. self-consistently determined. A unique manifold of solution exists (see "theorem of uniqueness" in Pasetto et al. (2014)) that yields all the possible solutions. This differs from the MLT where the same solution is obtained with a degree of freedom (i.e. the mixing-length). Now, this extra degree of freedom is fixed by introducing another equation of motion for the convective layers (i.e. expansion in addition to the vertical motion). Finally, it is worth noting that in the SFC theory the convective energy transport of the energy occurs mainly by the expansion of the elements and less by their vertical motion.

\section{Results}

We present here an extended comparison between the standard MLT and the SFC theory. The results are obtained from solving the system of Eq.2.1 for each layer of a stellar atmosphere governed by the equations considered in Section 2.1.

\subsection{The model matching the Sun}

We consider the stellar track of Bertelli et al. (2008) best fiiting the present position of the Sun on the HRD e.g., $\log _{10}\left\{L / L_{\odot}, T_{\text {eff }}\right\} \cong\{0.000,3.762\}$ with standard chemical composition $\{X, Y\}=\{0.71,0.27\}$. The results are shown in Fig.1. In the same plot we

$\dagger$ The present formulation, however, cannot be applied to convective overshooting for which a suitable approach must be developed. 

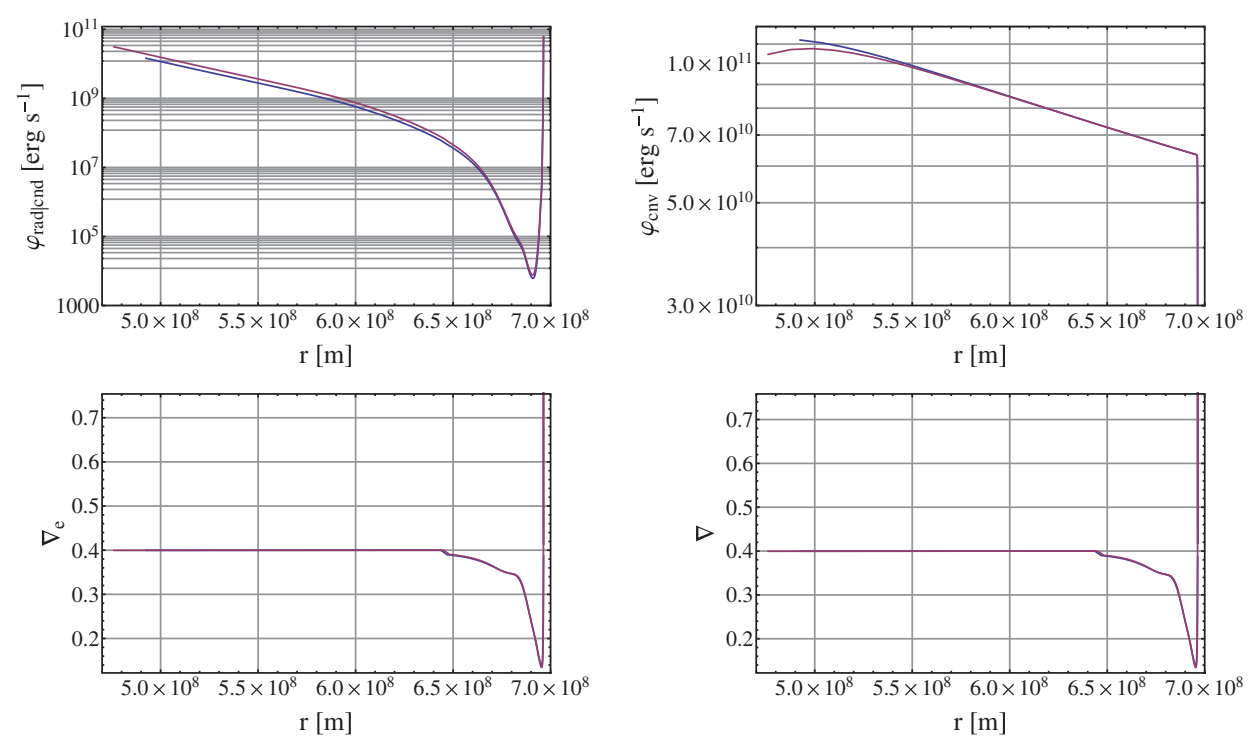

Figure 1. Solar fluxes and temperature gradient profiles for the internal convective stratification of the star. The upper panels show the expectation for $\varphi_{\mathrm{rad} \mid \mathrm{cnd}}$ on the left and $\varphi_{\mathrm{cnv}}$ on the right. Blue refers to our theory, purple to the MLT.

show also the predictions of the MLT with $\Lambda_{m}=1.65$ (the MLT is according to the version presented in Kippenhahn et al. (2012)), so that comparison between SFC theory and MLT is possible. Both the temperature gradients $\nabla$ and $\nabla_{e}$ and fluxes $\varphi_{\text {rad } \mid c n d}$ and $\varphi_{\mathrm{cnv}}$ predicted by SFC theory and MLT are in mutual agreement. A slightly larger convective zone is predicted by SFC theory the systematically lower radiative flux is balanced by higher convective flux.

\section{Conclusions}

We have presented here the first results of the integration of stellar atmospheres with SFC theory developed in Pasetto et al. (2014). We have set up a numerical code to systematically integrate as function of time and position the equations presented in Pasetto et al. (2014) and have run it in parallel with the standard MLT. All the results achieved by MLT are successfully recovered by the SFC theory without making use of any adjustable free-parameter. We argue that the new theory despite its linear formulation is able to capture the essence of the convection in the stellar atmospheric layers in a simple manner.

Furthermore, the SFC theory has a predictive potential that descriptive analyses of numerical simulations still miss. To be able to generate numerical simulations with milliondegrees of freedoms does notautomatically mean that we fully understand them. An emblematic example of these problems has recently been discussed by Arnett et al. (2015) where the authors fail to close the equations suggested their hydrodynamic simulations. Their ultimate goal is to search 1D theory based on 3D simulations passing through a $2 \mathrm{D}$ intermediate stage. We dare to claim here that a $1 \mathrm{D}$, parameter-free theory already exist Pasetto et al. (2014) and that this is right trail to follow. 


\section{References}

Alongi, M., Bertelli, G., Bressan, A., \& Chiosi, C., 1991, A\&A, 244, 95

Arnett, W. D., Meakin, C., Viallet, M., Campbell, S. W., Lattanzio, J., \& Moćak M., 2015, ArXiv e-prints

Bertelli, G., Bressan, A., Chiosi, C., Ng, Y. K., \& Ortolani, S., 1994, Memorie della Societ Astronomia Italiana, 65, 689

Bertelli, G., Bressan, A., Chiosi, C., Ng, Y. K., \& Ortolani, S., 1995, A\&A, 301, 381

Bertelli, G., Girardi, L., Marigo, P., \& Nasi, E., 2008, A\& $A, 484,815$

Bertelli, G. \& Nasi, E., 2001, AJ, 121, 1013

Bertelli, G., Nasi, E., Girardi, L., Chiosi, C., Zoccali, M., \& Gallart, C., 2003, AJ, 125, 770

Biermann, L., 1951, Zeitschrift fur Astrophysik, 28, 304

Böhm-Vitense E., 1958, Zeitschrift fur Astrophysik, 46, 108

Bressan, A. G., Chiosi, C., \& Bertelli, G., 1981, A\& A, 102, 25

Chiosi, C. \& Summa, C., 1970, Ap\&SSS, 8, 478

Deng, L., Bressan, A., \& Chiosi, C., 1996a, A $ध A$, 313, 145

Deng, L., Bressan, A., \& Chiosi, C., 1996b, A\&A A, 313, 159

Hofmeister, E., Kippenhahn, R., \& Weigert, A., 1964, Zeitschrift fur Astrophysik, 59, 215

Kippenhahn, R., Weigert, A., \& Weiss, A., 2012, Stellar Structure and Evolution

Pasetto, S., Chiosi, C., Cropper, M., \& Grebel, E. K., 2014, MNRAS, 445, 3592

Salasnich, B., Bressan, A., \& Chiosi, C., 1999, A\&A, 342, 131

Tuteja, G. S., Khattar, D., Chakraborty, B. B., \& Bansal, S., 2010, Int. J. Contemp. Math. Sciences, 5, 1065

Weiss, A. \& Schlattl, H., 2008, ApESSS, 316, 99 